\title{
Splicing regulator SLU7 is essential for maintaining liver homeostasis
}

\author{
María Elizalde,, Raquel Urtasun,, ${ }^{1}$ María Azkona, ${ }^{1}$ María U. Latasa,, Saioa Goñi, ${ }^{1}$ \\ Oihane García-Irigoyen, ${ }^{1}$ Iker Uriarte,, ${ }^{2}$ Victor Segura, ${ }^{1}$ María Collantes, ${ }^{3}$ Mariana Di Scala, ${ }^{1}$ \\ Amaia Lujambio, ${ }^{4,5}$ Jesús Prieto,, ${ }^{1,2}$ Matías A. Ávila,, ${ }^{1,2}$ and Carmen Berasain ${ }^{1,2}$
}

\begin{abstract}
${ }^{1}$ Division of Hepatology and Gene Therapy, Centro de Investigación Médica Aplicada (CIMA), Universidad de Navarra, Pamplona, Spain. ${ }^{2}$ CIBERehd, Instituto de Salud Carlos III, Madrid, Spain. ${ }^{3}$ Small Animal Imaging Research Unit, CIMA, Clínica Universidad de Navarra, Pamplona, Spain. ${ }^{4}$ Cold Spring Harbor Laboratory, Cold Spring Harbor, New York, USA. ${ }^{5}$ Memorial Sloan-Kettering Cancer Center, New York, New York, USA.
\end{abstract}

\begin{abstract}
A precise equilibrium between cellular differentiation and proliferation is fundamental for tissue homeostasis. Maintaining this balance is particularly important for the liver, a highly differentiated organ with systemic metabolic functions that is endowed with unparalleled regenerative potential. Carcinogenesis in the liver develops as the result of hepatocellular de-differentiation and uncontrolled proliferation. Here, we identified $S L U 7$, which encodes a pre-mRNA splicing regulator that is inhibited in hepatocarcinoma, as a pivotal gene for hepatocellular homeostasis. SLU7 knockdown in human liver cells and mouse liver resulted in profound changes in pre-mRNA splicing and gene expression, leading to impaired glucose and lipid metabolism, refractoriness to key metabolic hormones, and reversion to a fetal-like gene expression pattern. Additionally, loss of SLU7 also increased hepatocellular proliferation and induced a switch to a tumor-like glycolytic phenotype. $S l u 7$ governed the splicing and/or expression of multiple genes essential for hepatocellular differentiation, including serine/arginine-rich splicing factor $3(S r s f 3)$ and hepatocyte nuclear factor $4 \alpha(H n f 4 \alpha)$, and was critical for cAMP-regulated gene transcription. Together, out data indicate that SLU7 is central regulator of hepatocyte identity and quiescence.
\end{abstract}

\section{Introduction}

The liver performs a variety of unique functions essential for the preservation of homeostasis, including glucose and lipid metabolism, xenobiotic detoxification, and serum protein synthesis. Most of these roles are performed by the hepatocyte, a quiescent and highly differentiated cell expressing a complement of enabling genes (1). The liver's central position in systemic metabolism implies a prominent exposure to noxious stimuli derived from environmental toxicants, alcohol, viruses, and dietary habits, the principal causes of liver disease (2). To cope with these challenges, the liver has developed a remarkable and tightly controlled regenerative capacity based on the proliferation of hepatocytes in response to parenchymal loss (3). However, persisting injury may lead to the development of liver cirrhosis, a condition characterized by extracellular matrix accumulation and regenerative hepatocellular nodules (4). Another important hallmark of the chronically injured liver is progressive loss of function due to a gradual decrease in the expression of hepatospecific genes (5-8). These changes are often accompanied by unrestrained cell proliferation and reactivation of genes characteristic of the fetal hepatocyte $(7,9,10)$.

Human and experimental studies indicate that hepatocellular dedifferentiation may be linked to the development of hepatocellular carcinoma (HCC), a most serious complication of chronic liver injury and cirrhosis (11). Clinical findings demonstrated that the degree of differentiation of HCC tissues positively correlated with patient survival (12). More recently, analyses of gene expression profiles in HCCs (13) and peritumoral cirrhotic liver tissues (14)

Authorship note: Jesús Prieto, Matías A. Ávila, and Carmen Berasain are co-senior authors.

Conflict of interest: The authors have declared that no conflict of interest exists. Citation for this article: J Clin Invest. 2014;124(7):2909-2920. doi:10.1172/JCI74382. provided molecular confirmation of these histological observations, linking expression patterns indicative of fetal and dedifferentiated status with poor patient prognosis. However, little is known about the mechanisms driving hepatocellular dedifferentiation during chronic liver disease and tumor development. Impaired expression of a limited set of genes generally known as liver-enriched transcription factors or hepatocyte nuclear factors (HNFs) (1) could play a fundamental role. The best characterized is the nuclear receptor $\mathrm{HNF} 4 \alpha$, a transcriptional regulator involved in embryonic liver development and in the maintenance of the differentiated phenotype of adult hepatocytes $(15,16)$. HNF $4 \alpha$ expression is reduced in liver cirrhosis (7), contributing to experimental hepatocarcinogenesis (17). A recent study demonstrated that the pre-mRNA splicing regulator serine/arginine-rich splicing factor 3 (SRSF3) was also essential for hepatic maturation and metabolic function in mice (18), indicating that preservation of hepatocellular identity was not restricted solely to HNFs. Altered expression and activity of splicing factors has been lately reported in different tumor types, including HCC (19-21). These observations highlight the importance of accurate pre-mRNA splicing for liver homeostasis and the implication of deranged splicing in disease progression (22). Recently we reported that SLU7, a splicing factor critical for the correct selection of the $3^{\prime}$ splice site (23), was downregulated in human cirrhotic liver and HCC and that loss of SLU7 resulted in the aberrant production of a protumorigenic splice variant of the P73 tumor suppressor (24). Here we identified SLU7 as a fundamental gene for hepatocellular differentiation and quiescence. $\operatorname{Slu} 7$ knockdown in adult mouse liver gave raise to a striking phenotype, encompassing impaired glucose and lipid metabolism, loss of response to key metabolic hormones, and reversion to a fetal-like gene expression pattern. Moreover, Slu 7 downregulation resulted in hepatocellular proliferation and a metabolic switch to a glycolytic 
phenotype reminiscent of that found in tumors (25-27). Mechanistically, SLU7 modulated the splicing and expression of genes essential for the preservation of the hepatocyte identity, including $\operatorname{Srsf} 3$ and $H n f 4 \alpha$, and we identified SLU7 as a key transcriptional regulator in the cAMP signaling pathway.

\section{Results}

Knockdown of SLU7 induces profound changes in pre-mRNA splicing and gene expression in buman HCC cells. To investigate the role of SLU7 in human liver cell biology, we knocked down SLU7 expression in $\mathrm{PLC} / \mathrm{PRF} / 5$ HCC cells and performed microarray analyses of exon utilization and gene expression. We detected 590 splice events (504 exons upregulated, 86 downregulated). Ingenuity Pathway Analysis of data according to cellular functions revealed that most of these altered exon use events occurred in genes involved in lipid and carbohydrate metabolism, cell cycle, proliferation, and survival pathways (Figure 1A and Supplemental Table 1; supplemental material available online with this article; doi:10.1172/ JCI74382DS1). Interestingly, the most represented category was that of genes implicated in RNA posttranslational modification (Figure 1A). Gene expression microarray data were consistent with our observations in the exon utilization microarray: among the 243 genes with altered expression (85 upregulated, 158 downregulated), the majority of changes occurred in genes implicated in carbohydrate and lipid metabolism, cell proliferation and survival, and posttranscriptional RNA modification (Figure 1B and Supplemental Table 2). Most variations in exon utilization and gene expression affected metabolic genes typical of the mature hepatocyte phenotype, which suggests that SLU7 plays an important role in hepatocellular differentiation. This hypothesis was reinforced by our finding of altered SRSF3 pre-mRNA splicing upon SLU7 silencing (Supplemental Table 1). In view of the key role of SRSF3 in liver function (18), we further explored the influence of SLU7 on SRSF3 pre-mRNA splicing. SRSF3 pre-mRNA alternative splicing generates the full-length isoform lacking exon 4 (Iso1) and an alternative isoform including exon 4 (Iso2) (Figure 1C). As occurs for all SR genes, alternative isoforms like SRSF3 Iso2 contain a poison cassette exon introducing an in-frame premature termination codon promoting SR transcript degradation (28). SLU7 knockdown triggered an increase in the SRSF3 Iso2/Iso1 ratio in several human hepatoma cell lines (Figure 1, C and D). This response was validated in the nontransformed human liver cell line HepaRG (Figure 1E and ref. 29). Consistently, SLU7 overexpression in HepaRG cells had the opposite effect on the SRSF3 Iso2/Iso1 ratio (Figure 1F). SRSF3 expression in human liver tissues has not previously been examined. SRSF3 mRNA levels were reduced in HCC samples compared with control tissues, and a tendency toward reduced levels (without reaching statistical significance) was noted in cirrhotic liver tissues (Figure 1G). Interestingly, expression of SRSF3 and SLU7 directly correlated when all liver tissue samples were collectively analyzed (Figure $1 \mathrm{H}$ ).

SLU7 defines a liver-specific gene expression pattern in buman hepatocytes and is a developmentally regulated gene in the mouse liver. Next, we directly analyzed the influence of SLU7 on relevant genes characteristic of the hepatocellular identity. Upon SLU7 knockdown in HepaRG cells, the expression of albumin $(A L B)$, $H N F 4 \alpha$, and methionine-adenosyltransferase 1A (MAT1A) (30) was significantly inhibited, while the oncofetal marker $H 19$ (31) was induced (Figure 2A). Moreover, the ratio between the CYP4F3 splice variants $C Y P 4 F 3 A$ and $C Y P 4 F 3 B$ was markedly increased
(Figure 2A), also indicative of hepatocellular dedifferentiation (32). Reduced CYP4F3B mRNA levels were also observed in our expression microarray analysis (Supplemental Table 2). Complementarily, SLU7 overexpression increased HNF4 $\alpha$ and MAT1A expression (Figure 2B). These effects were reproduced in HepG2 cells (Supplemental Figure 1).

During mouse liver development, $\operatorname{Slu} 7 \mathrm{mRNA}$ followed a temporal expression pattern (Figure 2C), similar to that of $H n f 4 \alpha$ and Mat1a (33), and opposite that displayed by Wilms' tumor suppressor gene 1 (Wt1), which is expressed in fetal but not in adult hepatocytes and is reactivated in liver cirrhosis and $\operatorname{HCC}(7,8)$. These findings were corroborated at the protein level (Figure 2D). Immunohistochemical analysis of SLU7 in adult mouse liver showed a predominantly hepatocellular and nuclear pattern (Figure 2E).

Knockdown of Slu 7 in mouse liver disrupts the gene expression pattern characteristic of hepatocellular identity and markedly impairs physiological metabolic responses. We next assessed the role of SLU7 in vivo. Hepatocellular Slu 7 expression was downregulated using an adeno-associated viral vector expressing a Slu7-specific shRNA regulated by a hepatocyte-specific promoter (referred to herein as AAV-shSLU7; Figure 3, A and B). Importantly, the effect of reduced SLU7 expression on SRSF3 alternative splicing that we had observed in vitro was reproduced in mouse livers (Figure 3C). Moreover, Slu 7 knockdown markedly reduced $A l b, H n f 1 \alpha$, and $H n f 4 \alpha$ gene expression and significantly altered the expression of genes involved in hepatic lipid, cholesterol, and glucose metabolism (Figure 3D). Importantly, these effects were accompanied by induction of the oncofetal markers alphafetoprotein $(A f p)$ and $W t 1$ and of activating transcription factor 3 (Atf 3 ) and P53, stress-responsive genes in the liver (Figure 3D and refs. 34, 35). The specificity of the effects of $\operatorname{Slu} 7$ on liver gene expression was better illustrated by reciprocal changes in specific isozymes transcripts. Slu 7 knockdown resulted in downregulation of Mat1a, glutaminase 2 (Gls2), glucokinase (Gck), and L-pyruvate kinase $(L p k)$ and concomitant upregulation of Mat2a, Gls1, hexokinase 2 (Hk2), and pyruvate kinase $\mathrm{m} 2(P k m 2)$, together with a switch in the expression of $H n f 4 \alpha$ isoforms derived from the P1 and P2 gene promoters and in the ratio of Insr A and B splice variants (Figure $3 \mathrm{E}$ and Supplemental Figure 2). The isozymes, $H n f 4 \alpha \mathrm{P} 2$-derived isoforms, and splice variants favored upon Slu 7 downregulation were characteristic of the fetal and/or transformed hepatocyte, which exert profound effects on metabolism (26, 30, 36-40).

In association with these changes, $\operatorname{Slu} 7$ downregulation strongly influenced serum levels of glucose, cholesterol, and triglycerides and reduced intrahepatic glycogen stores (Figure 3F and Supplemental Figure 3). These effects were not related to liver damage, as only a mild elevation in serum alanine aminotransferase (ALT) was observed ( $98 \pm 8$ vs. $69 \pm 5 \mathrm{UI} / 1, P=0.008, n=31$ ), while aspartate aminotransferase (AST) remained unaltered (169 \pm 14 vs. $163 \pm 13$ $\mathrm{UI} / 1, P=0.88, n=31$ ). No hepatic histopathological alterations were found in Slu7-depleted mice (data not shown).

The liver plays a central role in blood glucose homeostasis, breaking down glycogen stores in the postabsorptive phase and synthesizing glucose as fasting progresses (36). In addition to depleted glycogen stores, Slu 7 knockdown induced fasting hypoglycemia (96 \pm 3 vs. $112 \pm 3 \mathrm{mg} / \mathrm{dl}$ blood glucose, $P<0.0025$, $n=18)$. These findings prompted us to examine the expression of phosphoenolpyruvate carboxykinase (Pepck) and glucose6-phosphatase $(G 6 p c)$, which are the 2 rate-limiting enzymes in gluconeogenesis, and of glucose-6-phosphate transporter 
A Alternative splicing

Lipid metabolism

Cell cycle

Carbohydrate metabolism

Cellular growth and proliferation

Cell death and survival

Gene expression

RNA post-transcriptional modification

C

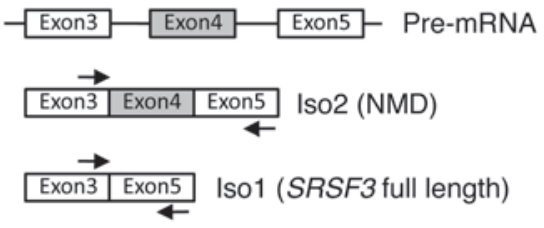

SiGL siSLU7

E
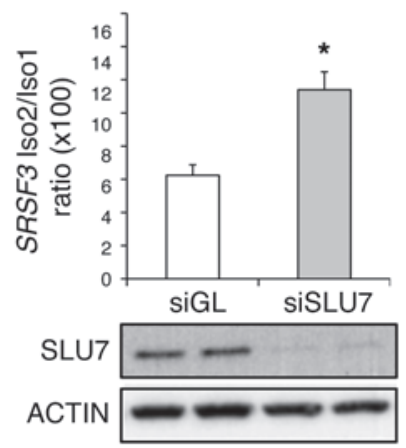

G

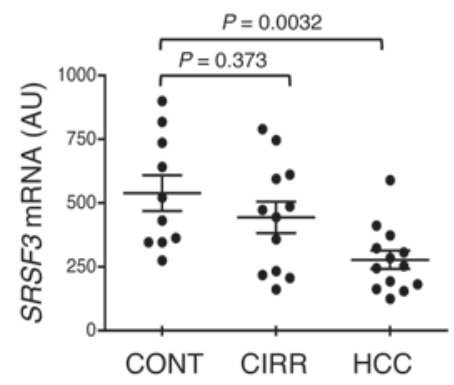

B Differential expression

RNA post-transcriptional

$$
\text { modification }
$$

Lipid metabolism

Cellular growth and proliferation

$$
\text { Cell cycle }
$$

Cell death and survival

Carbohydrate metabolism

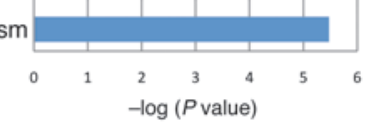

D

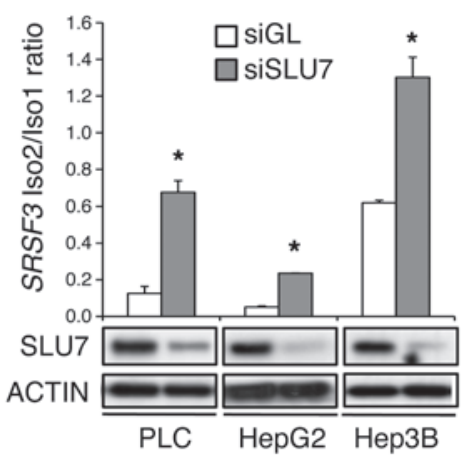

F
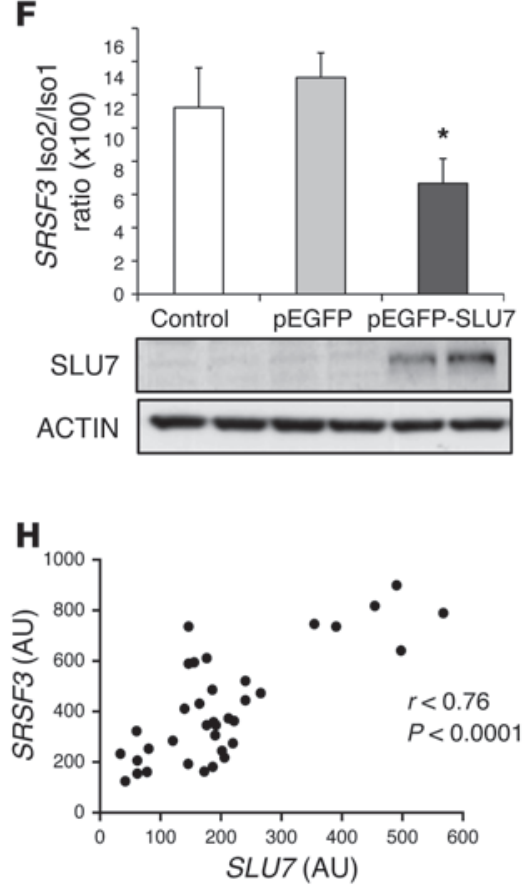

\section{Figure 1}

SLU7 strongly influences the gene expression profile of human liver cell lines and modulates SRSF3 splicing. (A and B) Top categories of genes undergoing changes in (A) splicing events and (B) expression in PLC/PRF/5 cells induced by SLU7 knockdown, classified in pathways according to Ingenuity Pathway Analysis. (C) SLU7 downregulation affected SRSF3 alternative splicing, promoting the generation of Iso2 targeted for nonsense-mediated decay (NMD). Bottom: Representative gel analyzing SRSF3 Iso1 and Iso2 PCR products in cells transfected with siSLU7 or siGL control. (D) qPCR analysis of SRSF3 Iso2/lso1 ratio in PLC/PRF/5, HepG2, and Hep3B cells transfected with siGL or siSLU7. Representative Western blots of SLU7 and ACTIN protein levels are also shown. ${ }^{*} P<0.05$ vs. siGL. (E and F) $\mathrm{qPCR}$ analysis of $S R S F 3$ Iso2/Iso1 ratio (E) in HepaRG cells upon SLU7 knockdown and $(F)$ in untransfected HepaRG cells (Control) or cells transfected with control vector ( $p E G F P)$ or SLU7 expression vector (pEGFP-SLU7). Bottom: Representative Western blot analysis of SLU7 and ACTIN protein levels. ${ }^{*} P<0.05$ vs. siGL or pEGFP. (G) SRSF3 expression, analyzed by qPCR, in human liver tissues from control, cirrhotic, and HCC tissue samples. (H) Spearman correlation analysis of SLU7 and SRSF3 expression, analyzed by qPCR, in all groups of human liver tissue samples. 
A

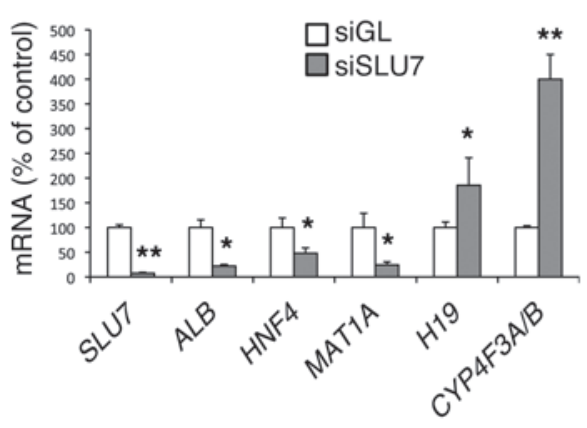

c

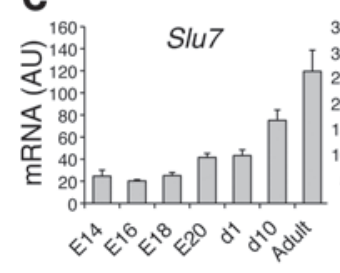

D

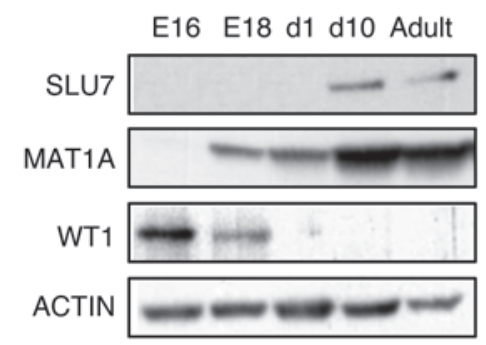

B
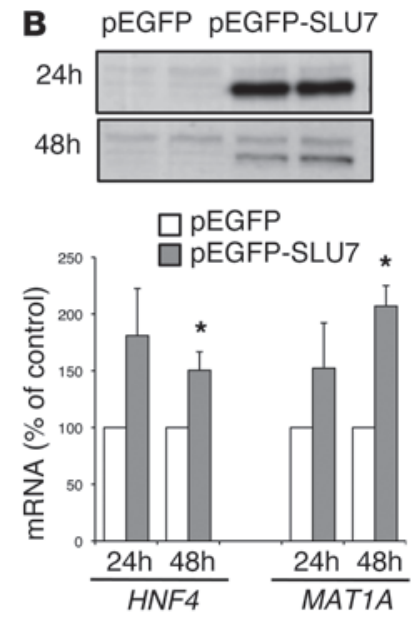
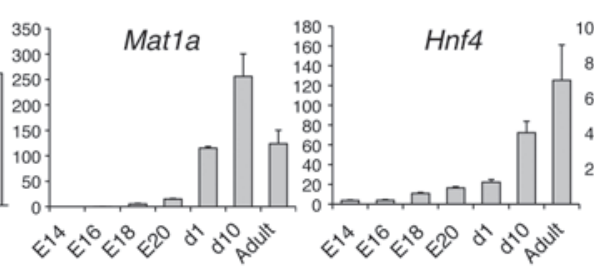

\section{E}

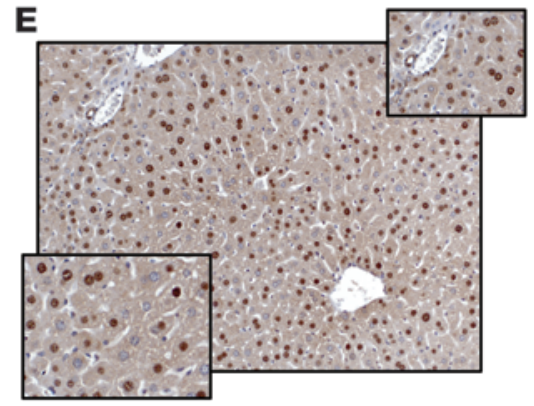

Figure 2

SLU7 modulates the expression of adult and fetal markers in human liver cells and is developmentally regulated in mice. (A) qPCR analysis of the expression of adult ( $A L B, H N F 4 \alpha$, and MAT1A) and fetal (H19 and CYP4F3A/B splice variant ratio) marker genes in HepaRG cells transfected with siGL or siSLU7. ${ }^{*} P<0.05,{ }^{* \star} P<0.01$ vs. siGL. (B) pEGFP-SLU7-mediated SLU7 overexpression in HepaRG cells promoted expression of the hepatocellular markers HNF4 and MAT1A. ${ }^{*} P<0.05$ vs. pEGFP. Top: Representative Western blot analyses of SLU7 protein levels at the indicated time points after transfection. (C) qPCR analysis of Slu7, Mat1a, Hnf4 $\alpha$, and Wt1 expression in fetal and postnatal mouse liver. (D) Representative Western blot analyses of SLU7, MAT1A, and WT1 protein levels in fetal and postnatal mouse liver. ACTIN levels are shown as control. (E) Representative images of immunohistochemical detection of SLU7 in adult mouse liver. Original magnification, $\times 20 ; \times 40$ (insets).

(G6pt), which is essential in the final steps of gluconeogenesis (36). Slu7-depleted mice showed impaired fasting-induced Pepck and G6pc upregulation and constitutive reduction in G6pt mRNA (Figure 4A). These observations were consistent with diminished de novo hepatic glucose production in a pyruvate tolerance test and a lower hyperglycemic response after glucagon challenge (Figure 4, B and C). Congruently, an enhanced hyperglycemic response was observed when a pyruvate tolerance test was performed in SLU7-overexpressing mice (infected with SLU7-expressing AAVs [AAV-SLU7]; Supplemental Figure 4, A and B). Together, these findings indicated that $\operatorname{Slu} 7$ is necessary for liver glucose production.
To gain mechanistic insights into the effects of SLU7 on gluconeogenesis, we directly examined its involvement in the regulation of $P E P C K$, which was identified as the second most downregulated gene in our microarray analysis (Supplemental Table 2). Basal levels of PEPCK gene expression were reduced upon $S L U 7$ knockdown in human liver cells (Figure 4, D and E). This effect was markedly attenuated by the concomitant overexpression of HNF $4 \alpha$ (Supplemental Figure $4 C$ ), which suggests that under resting conditions, SLU7-dependent PEPCK expression could be mediated to a great extent through $\mathrm{HNF} 4 \alpha$. We also found that cAMP-induced PEPCK protein and mRNA levels were strongly dependent on SLU7 expression in human liver cells and were also stimulated by forskolin (Figure 4, D and E, and Supplemental Figure 4D). This finding was extended to NR4A2 (also known as NURR1), another prototypical cAMP-regulated gene $(41,42)$, while ACTIN mRNA remained unaffected (Figure 4E). cAMP-induced transcription of PEPCK and NR4A2 genes is critically regulated by cAMP response elements (CREs) in their proximal promoters (42, 43). cAMP triggers phosphorylation of CRE-binding protein (CREB), facilitating its association with the coactivator CREB-binding protein (CBP). CBP is essential for RNA polymerase II (RNAPol II) recruitment to drive transcription of cAMP-responsive genes like PEPCK and NR4A2 (41, 42, 44, 45). Using ChIP assays, we demonstrated the association of SLU7 with PEPCK and NR4A2 proximal promoters (Figure 4F and Supplemental Figure 4E). Moreover, SLU7 was required for the association of CBP and RNA-Pol II with PEPCK and NR4A2 promoters, under both basal and cAMP-stimulated conditions (Figure 4G). Therefore, SLU7 seems essential for the assembly of the basic and cAMP-triggered transcriptional complex at the promoter of cAMPresponsive genes. A direct implication of SLU7 in this process is supported by our finding of coimmunoprecipitation of phosphorylated CREB (P-CREB), CBP, and RNA-Pol II with SLU7 (Figure 4H). Given that glucagon stimulates the gluconeogenic program through the cAMP/CREB signaling pathway (45), these observations contribute to explain our in vivo findings.

Lipogenesis is also a fundamental metabolic liver function. We observed that $\operatorname{Sl} 7$ knockdown markedly reduced the basal expression of key lipogenic genes (Figure 3D). The lipogenic genetic program is tightly controlled by nutrients and hormones like insulin, being physiologically modulated in response to diet (46). Slu 7 expression was also regulated in mouse liver upon fasting and refeeding, 

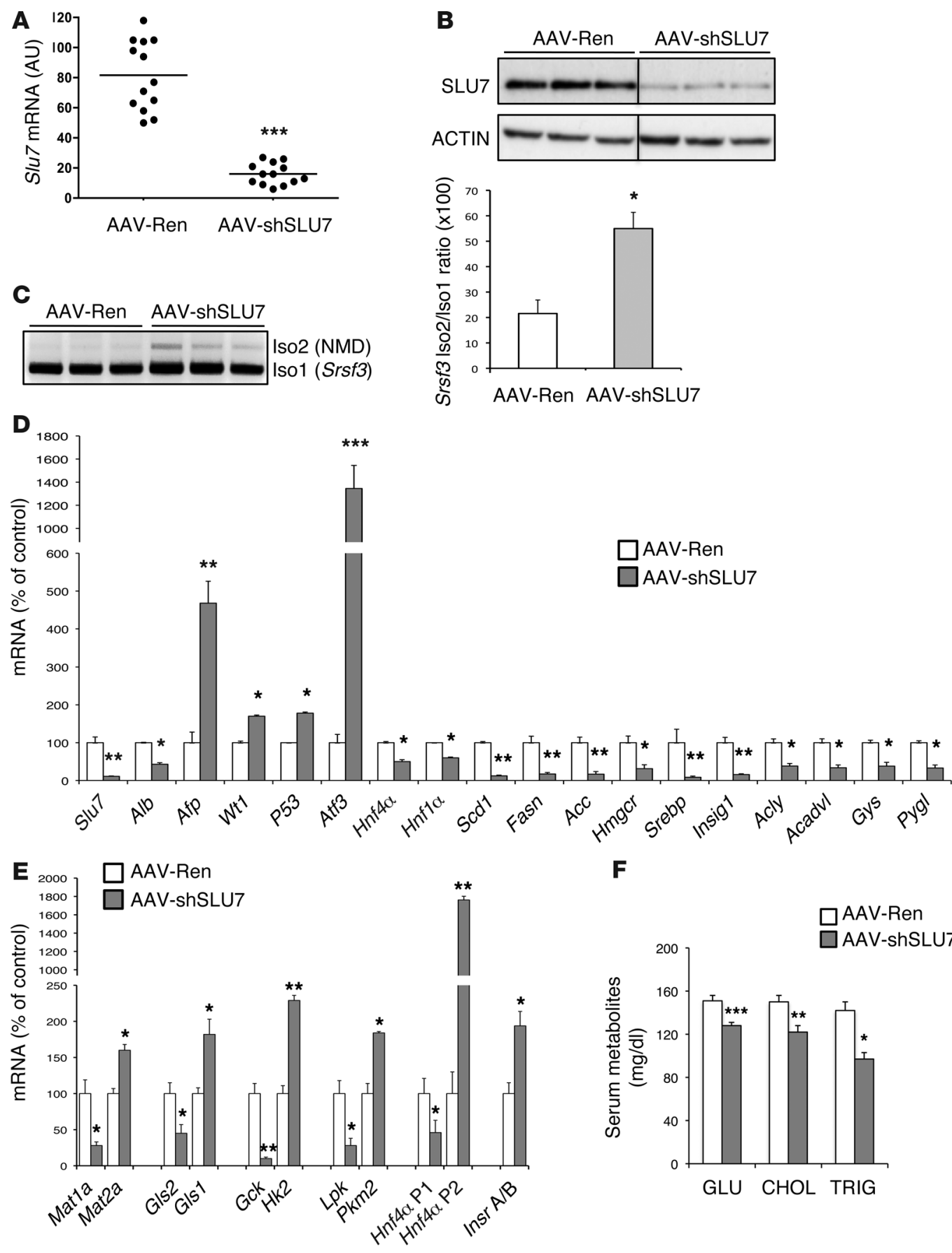

$\mathbf{F}$
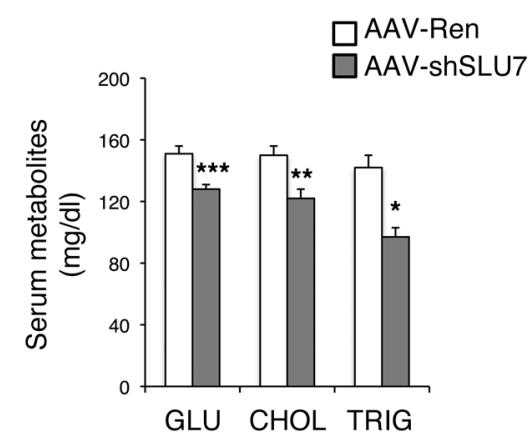

Figure 3

SLU7 is required for the preservation of the genetic program characteristic of the differentiated, quiescent, and metabolically functional liver. (A) qPCR analysis of S/u7 expression in livers of mice infected with AAV-shSLU7 or control (AAV-Ren). ${ }^{* * *} P<0.001$. (B) Representative Western blot analysis of SLU7 protein in liver tissues. ACTIN levels are shown as control. Lanes were run on the same gel but were noncontiguous. (C) Effect of Slu7 knockdown in mouse liver on Srsf3 alternative splicing. Left: Representative gel analyzing Srsf3 Iso1 and Iso2 RT-PCR products. Right: qPCR analysis of Srsf3 Iso2/lso1 ratio in livers. ${ }^{*} P<0.05$ vs. AAV-Ren. (D) qPCR analysis of expression of selected liver-enriched genes along with the fetal and proliferative hepatocyte markers Afp, Wt1, and Atf3. ${ }^{*} P<0.05,{ }^{\star \star} P<0.01,{ }^{* \star \star} P<0.001$ vs. AAV-Ren. (E) Liver-specific Slu7 knockdown induced a switch in the gene expression of metabolic enzymes, Hnf4 $\alpha$ P1/P2 promoter usage, and Insr splicing isoforms toward a pattern characteristic of the fetal and transformed hepatocyte. ${ }^{\star} P<0.05$, ${ }^{\star \star} P<0.01$ vs. AAV-Ren. (F) Liver-specific Slu7 knockdown influenced serum levels of glucose, cholesterol, and triglycerides. ${ }^{*} P<0.05,{ }^{* *} P<0.01,{ }^{* \star *} P<0.001$ vs. AAV-Ren. 

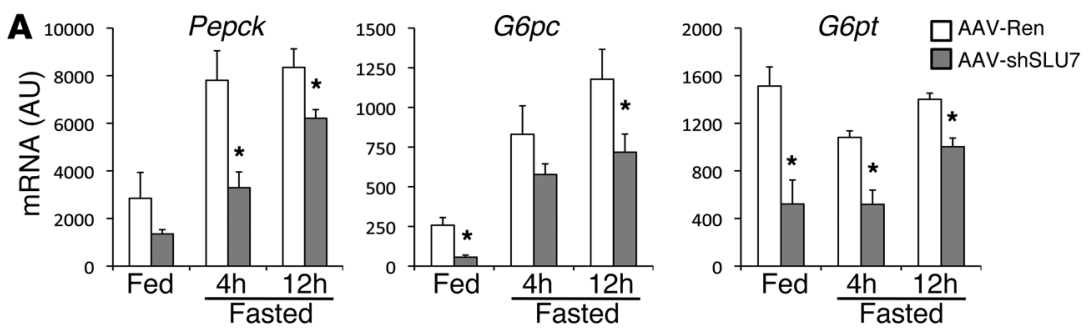

B
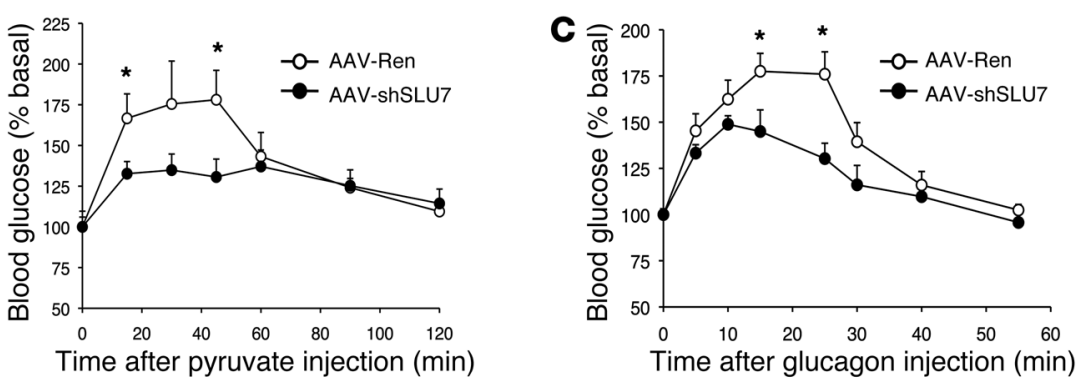

D
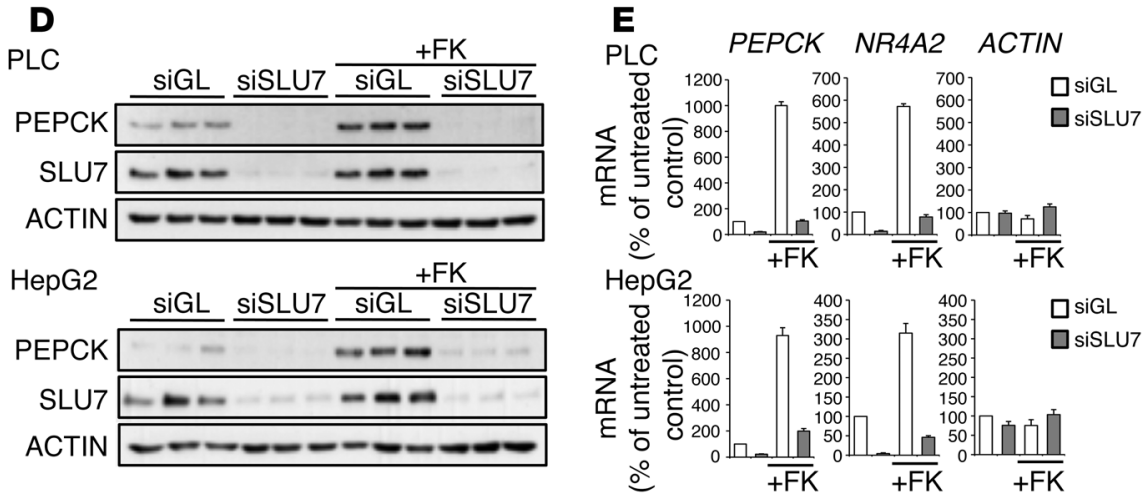

$\mathbf{F}$
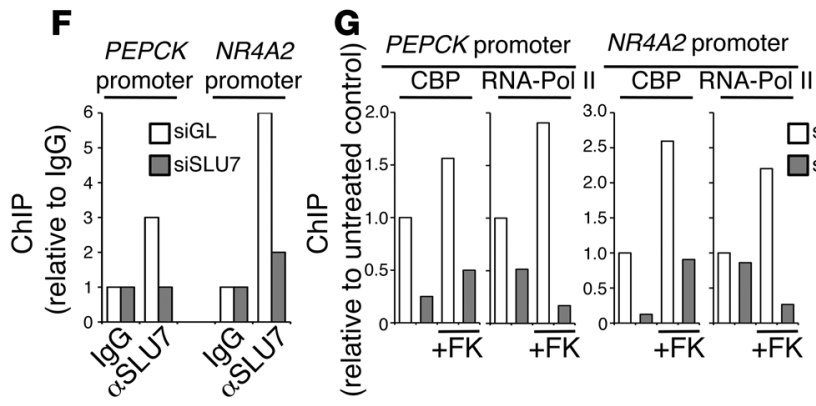

H

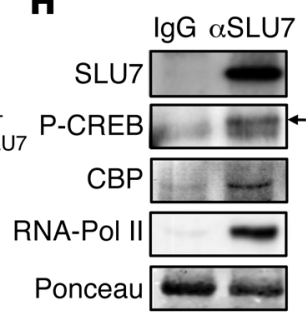

\section{Figure 4}

Metabolic effects of S/u7 depletion in mouse liver: SLU7 plays a central role in glucose production and is essential for cAMP-mediated gene expression. (A) qPCR analysis of Pepck, G6pc, and G6pt expression in fed and fasted AAVRen- and AAV-shSLU7-infected mice. ${ }^{*} P<0.05$ vs. AAV-Ren. (B and C) Hepatic glucose production after (B) pyruvate challenge and (C) glucagon challenge in AAVRen- and AAV-shSLU7-infected mice. ${ }^{*} P<0.05$ vs. AAV-shSLU7. (D and E) PLC/ $\mathrm{PRF} / 5$ and HepG2 cells were transfected with siGL or siSLU7. 48 hours later, cells were treated with $10 \mu \mathrm{M}$ forskolin (FK) for 4 hours, prior to analysis of PEPCK and/ or NR4A2 expression. (D) Representative Western blots of PEPCK, SLU7, and ACTIN control. (E) qPCR analysis of PEPCK, NR4A2, and ACTIN control. (F) ChIP assays using control (IgG) or antiSLU7 antibodies in siGL- or siSLU7-transfected PLC/PRF/5 cells analyzing PEPCK and NR4A2 proximal promoters. (G) ChIP assays of PEPCK and NR4A2 promoters in siGL- and siSLU7-transfected PLC/ PRF/5 cells. 48 hours after transfection, cells were treated with $10 \mu \mathrm{M}$ forskolin for 30 minutes, then subjected to ChIP with anti-CBP and anti-RNA-Pol II antibodies. (H) SLU7 coimmunoprecipitated with P-CREB (arrow), CBP, and RNA-Pol II in $\mathrm{PLC} / \mathrm{PRF} / 5$ cells. Lysates were immunoprecipitated with anti SLU7 antibodies or preimmune IgG. Representative Western blots of 3 experiments are shown. and tissue immunostaining revealed that predominantly pericentral expression became more apparent in the liver of fasted mice (Figure 5, A and B, and Supplemental Figure 5, A and B). Insulin may be a key factor in the nutritional control of $\operatorname{Slu} 7$ gene expression, as this hormone directly reduced Slu $7 \mathrm{mRNA}$ levels in primary mouse hepatocytes (Supplemental Figure 5C). Moreover, mice fed a highfat diet (HFD), a condition associated with insulin resistance, had increased basal levels of $\operatorname{Slu} 7$ expression in the liver and showed impaired $\operatorname{Slu} 7$ downregulation upon feeding (Supplemental Figure 5, D and E). Importantly, SLU7 played a fundamental role in the dietary regulation of Srebp1, the master transcription factor of lipogenesis. The prominent induction of Srebp1 expression elicited upon refeeding was lost in Slu7-depleted mouse liver (Figure 5, C and D). Consistently, the SREBP1 target genes Acly, Acc, Fasn, Hmgcr, and Insig1 did not respond to feeding in AAV-shSLU7-infected mice, while the expression of the non-SREBP1 target gene Insig $2 a$ decreased with feeding, as expected (47), in both mouse groups (Figure 5E).

Given the central role played by insulin in the dietary regulation of Srebp1 expression (47), we examined insulin sensitivity in AAVshSLU7-infected mice and found significant resistance to the action of the hormone (Figure 5F). The PI3K-AKT pathway is key in insulin-mediated Srebp1 expression (47). Consequently, liver AKT phosphorylation was clearly detected in control mice upon refeeding, but not in AAV-shSLU7-infected mice (Figure 5G). Consistent with this was the impaired phosphorylation of the downstream AKT target GSK3 $\alpha / \beta$ (Figure $5 \mathrm{G}$ and ref. 48 ). Together, these data are indicative of abnormal hepatic insulin signaling upon reduced SLU7. Slu7 knockdown profoundly altered the expression of developmentally regulated and liver-specific genes (Figure 3, D and E). Insr expression was also 
A

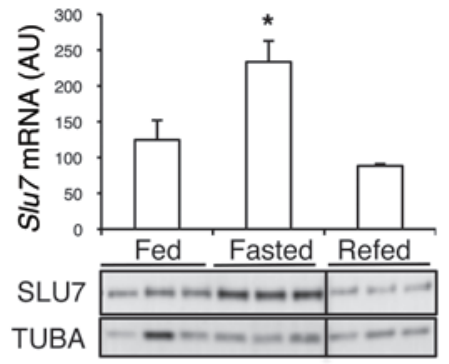

B

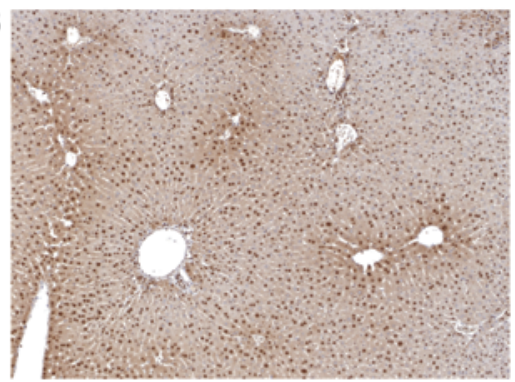

C

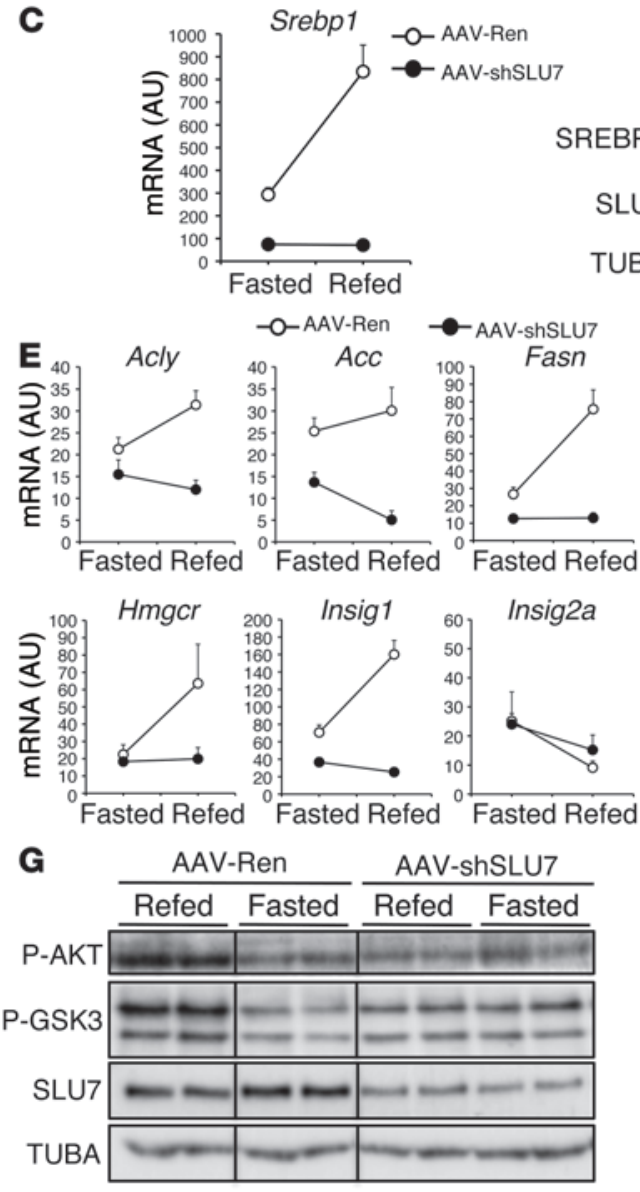

Srebp1
D

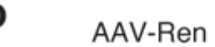

AAV-Ren

AAV-shSLU7

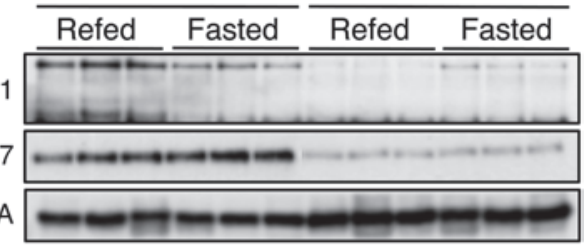

$\mathbf{F}$

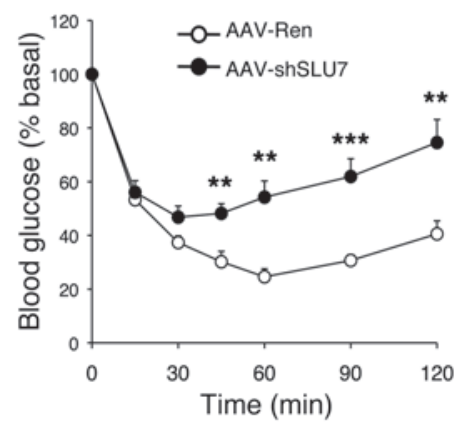

\section{Figure 5}

Slu7 depletion in mouse liver interferes with nutritional regulation of lipogenesis and blunts hepatic insulin responses. (A) S/u7 mRNA levels were analyzed by qPCR, and SLU7 protein levels by Western blot, in mice that were fed ad libitum, fasted for 14 hours, or fasted for 14 hours and subsequently refed for 4 hours. Lanes were run on the same gel but were noncontiguous. ${ }^{*} P<0.05$ vs. refed. (B) Immunohistochemical detection of SLU7 in liver tissues of mice fasted for 14 hours. Original magnification, $\times 20$. (C) qPCR analysis of Srebp1 expression in livers of AAV-Ren- and AAVshSLU7-infected mice upon fasting and refeeding. (D) Western blot analysis of SREBP1 protein in livers upon fasting and refeeding. SLU7 and tubulin (TUBA) are also shown. (E) qPCR analysis of the mRNA levels of SREBP1 target genes and the regulatory genes Insig1 and Insig2a in livers upon fasting and refeeding. (F) Blood glucose concentrations during insulin tolerance test. ${ }^{\star *} P<0.01,{ }^{* * \star} P<0.001$ vs. AAV-Ren. (G) Intracellular signaling in livers upon fasting and refeeding. Representative Western blots show phosphorylated AKT and GSK3 as well as SLU7 and tubulin. Lanes were run on the same gel but were noncontiguous. (H) Expression of Insr mRNA determined by $\mathrm{qPCR}$, and INSR protein ( $\beta$ chain) determined by Western blotting, in mouse livers. ${ }^{*} P<0.05$ vs. AAV-Ren. A representative blot is shown.

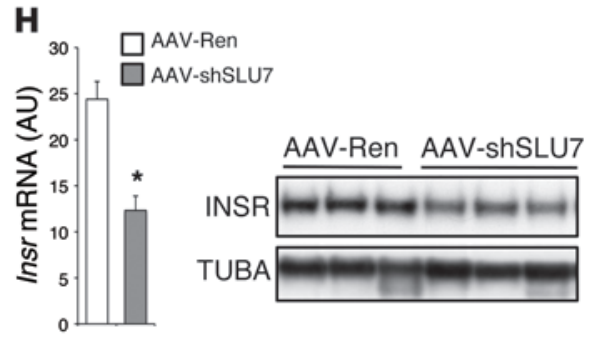

upregulated during liver development (Supplemental Figure 2 and ref. 49). We found that SLU7 knockdown had a strong influence on liver Insr expression (Figure 5H). Nevertheless, in addition to the regulation of the insulin signaling system, we cannot exclude a more direct effect of SLU7 on the regulation of SREBP1 expression. As we observed for gluconeogenic genes (Figure 4, A and D), basal expression of Srebp1 was significantly reduced upon $\operatorname{Slu} 7$ knockdown in vivo, as well as in human liver cells (Figure 5, C and D, and Supplemental Figure 5, F and G). Collectively, these findings indicate that SLU7 is required for the preservation of essential liver metabolic pathways and their hormonal regulation.

Reduced Slu 7 expression promotes aerobic glycolysis and liver growth. One of our most intriguing observations was the switch in the hepatic expression of the glycolytic isoenzymes $L p k$ to $P k m 2$ and Gck to Hk2 upon Slu7 knockdown (Figure 3E). PKM2 and HK2 favor aerobic glycolysis, a hallmark of transformed cells (27). Upregulation of PKM2 and HK2 proteins was confirmed in livers from Slu7-depleted mice (Figure 6A). We demonstrated that $\operatorname{Slu} 7$ downregulation resulted in significantly increased hepatic glucose uptake and catabolism, as demonstrated by intrahepatic lactate concentrations (Figure 6, B-D). The forced expression of PKM2 and HK2 activates hepatocellular growth in normal liver (39). We found that the cell cycle-related genes Foxm1, Nor1, and early growth response 1 (Egr1) and the cyclins Ccnd1, Ccna2, and $C c n b 2$ were all upregulated in $S l u 7$-depleted mouse livers and in SLU7-silenced HepG2 cells (Figure 7A and Supplemental Figure 6). Additionally, these mice showed increased expression of the HCCpromoting splicing protein heterogeneous nuclear ribonucleoprotein A1 (Hnrnpa1; Figure 7A and ref. 21), which was also identified in our microarray analysis (Supplemental Table 2). Overexpression 


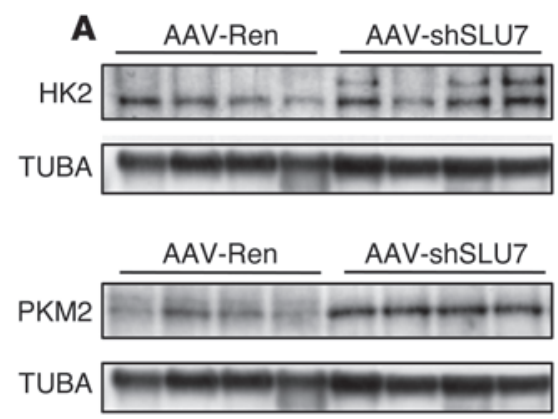

\section{$+$}
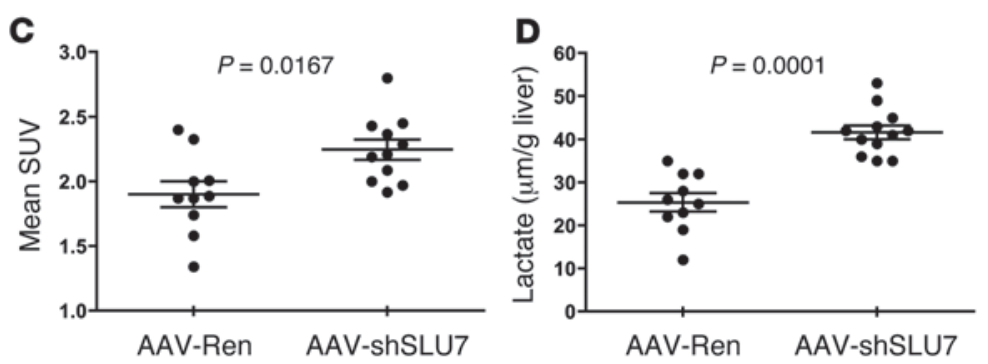

\section{Figure 6}

Slu7 knockdown promotes hepatic glycolytic gene expression and enhanced glucose uptake and metabolism. (A) Western blot analysis of HK2 (arrow) and PKM2 protein levels in livers of AAV-Ren- and AAV-shSLU7-infected mice. Tubulin is shown as control. Representative blots are shown. (B) Representative micro-PET images showing ${ }^{18} \mathrm{~F}-\mathrm{FDG}$ uptake. Dotted outlines denote livers. h, heart; b, bladder. (C) Quantification of ${ }^{18} \mathrm{~F}-\mathrm{FDG}$ uptake in the liver. (D) Quantification of intrahepatic lactate concentration.

of cell proliferation-related genes, including Myc (encoding c-Myc), and downregulation of $H n f 4 \alpha$ were also validated at the protein level (Figure 7B). Interestingly, the fast-migrating anti-HNF $4 \alpha-$ reactive bands we observed upon $\operatorname{Slu} 7$ knockdown may correspond to $H n f 4 \alpha$ P2 promoter-derived transcripts (50), which we found to be favored upon $\operatorname{Slu} 7$ downregulation (Figure 3E). Quite remarkably, these genetic changes translated into hepatocellular proliferation and a $23 \%$ increase in liver mass (Figure 7, C and D). To further establish the influence of SLU7 on hepatocellular proliferation, we evaluated its expression during liver regeneration after two-thirds partial hepatectomy (PH), as well as the effect of manipulating SLU7 levels on the expression of cell cycle-regulatory genes. We found a transient reduction in $\operatorname{Slu} 7$ gene expression shortly after liver resection (Supplemental Figure 7, A and B). Most interestingly, we observed enhanced expression of EGR1 and CCND1 after $\mathrm{PH}$ in the face of $\operatorname{Slu} 7$ knockdown (mice infected with AAV-shSLU7) and reduced levels of CCND1 after $\mathrm{PH}$ in mice overexpressing SLU7 (infected with AAV-SLU7) (Supplemental Figure 7, C and D).

\section{Discussion}

We have identified a fundamental new role for SLU7 in the preservation of liver function and hepatocellular quiescence. SLU7 downregulation in human liver cells impaired the expression of multiple genes characteristic of the mature and differentiated hepatocyte, inducing that of immature and proliferative cells. These findings were corroborated in healthy adult mice with liver-specific Slu7 knockdown, which developed a strong metabolic and proliferative hepatic phenotype. SLU7 is known as a pre-mRNA splicing regulator (51). However, the physiological role of SLU7 in vivo has not been addressed, previous works being restricted to in vitro

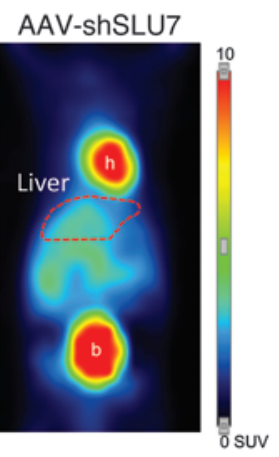

observations $(24,52)$. Our microarray analyses demonstrated SLU7 participation in the regulation of splicing and expression of genes implicated in RNA modification and liver metabolism. In particular, SLU7 knockdown modulated the splicing of SRSF3 not only in cultured cells, but also in normal mouse liver. SRSF3 is a RNA-binding protein and splice regulator, and its hepatocyte-specific deletion (SRSF3HKO mice) results in impaired hepatic maturation and disturbed lipid and glucose metabolism (18). We showed that SRSF3 expression was also reduced in human HCC, correlating significantly with that of SLU7 in healthy and diseased human liver tissues. This effect of SLU7 on SRSF3 expression may be of functional relevance, as the phenotype of the Slu7-depleted mice overlapped to a great extent with that of SRSF3HKO animals. Indeed, Slu 7 downregulation also resulted in decreased expression of liver-specific genes, including those involved in glucose and lipid metabolism and liver-enriched transcription factors, and in the upregulation of oncofetal markers. These findings suggested that the phenotypic alterations found upon $\operatorname{Slu} 7$ knockdown could be mediated by the concomitant downregulation of Srsf3. However, at variance with Slu7-depleted mice, SRSF3HKO animals did not show insulin resistance nor increased hepatocellular growth, and displayed extensive liver injury (18). This indicated that in addition to altering SRSF3 expression, SLU7 was also influencing other mechanisms essential for hepatocellular homeostasis. One key regulator of hepatocyte differentiation and function is $\operatorname{HNF} 4 \alpha(1,15)$. While SRSF3HKO mice showed no changes in HNF4 $\alpha$ levels (18), we found the expression of this liver-enriched transcription factor to be consistently reduced upon SLU7 knockdown, both in cultured cells and in mouse livers. $H N F 4 \alpha$ gene expression can be driven from 2 promoters, $\mathrm{P} 1$ and P2. P1 activity gives rise to the isoforms characteristic of the adult differentiated hepatocyte, while P2-derived transcripts are found in fetal and HCC cells and code for HNF $4 \alpha$ variants with less transactivation potential $(40,53,54)$. Remarkably, we found that $\operatorname{Slu} 7$ was essential for preserving the P1 and P2 promoter-driven $H n f 4 \alpha$ expression pattern of the adult liver. Hepatic HNF $4 \alpha$ expression is necessary for lipogenesis, cholesterol synthesis, and gluconeogenesis $(16,55)$. The concomitant reduction in SRSF3 and HNF4 $\alpha$ levels may explain, at least in part, the deranged expression of metabolic genes found in Slu7-depleted mice. Moreover, Hnf $4 \alpha$ downregulation may also help to explain the robust liver proliferative response detected in these mice. Hepatocyte-specific deletion of $H n f 4 \alpha$ results in hepatocellular dedifferentiation and proliferation and promotes chemically induced hepatocarcinogenesis. These responses were accompanied by upregulation of the promitogenic genes Egr1, Myc, and Ccnd1 (17, 56-58), which were also detected in Slu7-depleted mice. Interestingly, CCND1 can also interfere with HNF $4 \alpha$ transcriptional activity (57), while increased c-Myc levels modulate the switch in GLS1 and GLS2 expression (38) and, through the induction of hnRNPA1, can promote the expression of HK2 and PKM2 favoring glycolytic metabolism $(38,59)$. 
A

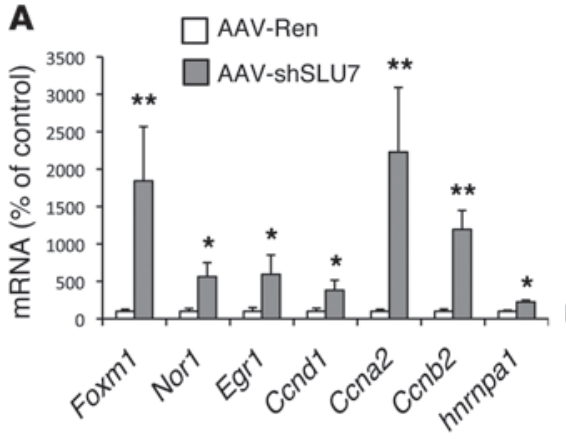

B

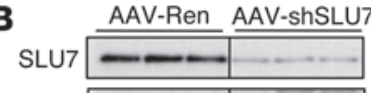

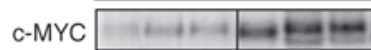

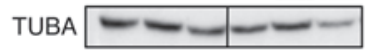

EGR1 DIELA

PCNA $-\infty-\infty$

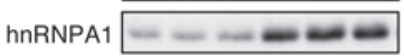

HNF4

TUBA

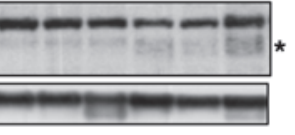

C
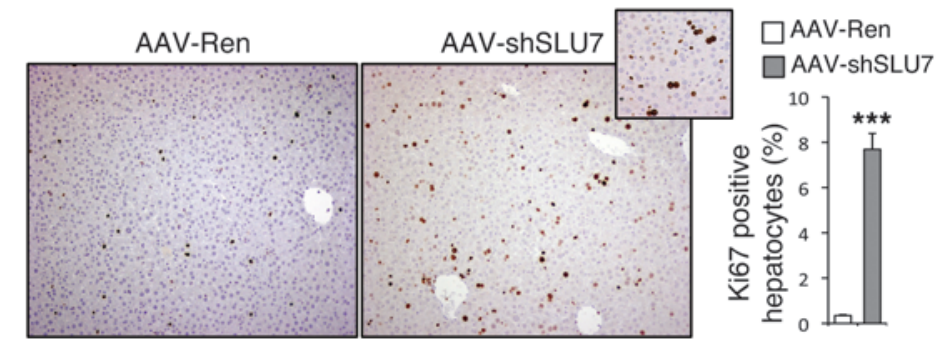

D

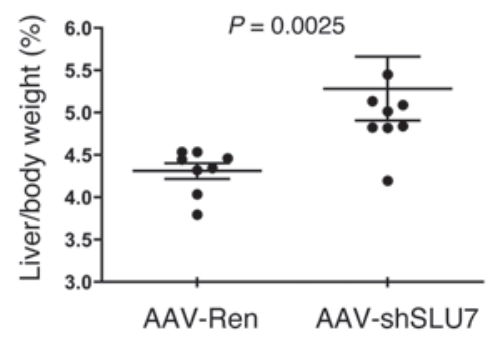

Figure 7

Slu7 knockdown triggers proliferation-related gene expression and promotes liver growth. (A) qPCR analysis of the expression of cell cycle- and proliferation-related genes in the livers of AAV-Ren- and AAV-shSLU7-infected mice. ${ }^{*} P<0.05$, ${ }^{* *} P<0.01$ vs. AAV-Ren. (B) Western blot analyses of selected proliferation-related proteins. SLU7 and tubulin are shown as controls. Asterisk indicates fast-migrating anti-HNF $4 \alpha$-reactive bands that may correspond to $H n f 4 \alpha$ P2 promoter-derived isoforms. Lanes were run on the same gel but were noncontiguous. (C) Left: Immunohistochemical analysis of Ki67 in liver sections. Data were acquired from livers corresponding to 12 (AAV-Ren) and 10 (AAV-shSLU7) mice; $10^{3}$ cells were counted per liver. Original magnification, $\times 10 ; \times 20$ (inset). Right: Quantification of Ki67-positive hepatocytes. ${ }^{* \star \star} P<0.001$. (D) Liver/body weight ratio.

The liver has an extraordinary regenerative capacity, with hepatocytes exhibiting almost unlimited proliferative potential (3). Characterization of the mechanisms governing the switch from quiescence to proliferation is a matter of great importance, not only for regenerative medicine, but also for its clear implications in hepatocarcinogenesis. Our current findings that HNF $4 \alpha$ levels were influenced by $\operatorname{Slu} 7$ gene expression, and that $\operatorname{Slu} 7$ knockdown triggered proliferation in an otherwise normal liver and facilitated the expression of cell cycle-driving genes after $\mathrm{PH}$, attest to the fundamental role of SLU7 in liver biology. The mechanisms behind $H N F 4 \alpha$ downregulation and P1/P2 promoter usage switch upon SLU7 knockdown are not currently known, although this effect could be partly mediated by the transcription factor WT1. This is supported by our previous observations demonstrating a potent negative effect of WT1 on $H N F 4 \alpha$ gene transcription in hepatocytes (7) and our current findings showing Wt1 upregulation upon $\operatorname{Slu} 7$ knockdown in mouse liver. Moreover, WT1 overexpression could also underlie the insulin resistance found in $\operatorname{Slu} 7$-deficient mice. Pari passu with SLU7, INSR expression was progressively induced during hepatocellular maturation, concomitantly with the decay of WT1 mRNA levels. This correlation and the previous identification of WT1 as a potent repressor of insulin gene promoter (60) lent support to this hypothesis.

One remarkable observation was the profound dysregulation of gluconeogenesis in Slu7-depleted mice. We not only found reduced expression of gluconeogenic genes in fed conditions, which could be attributed in part to low HNF4 $\alpha$ (61) and increased ATF3 expression (62), we also observed a marked impairment in the activation of the gluconeogenic transcriptional program by nutritional and hormonal stimuli. The cAMP signaling pathway plays a central role in this response, controlling the activity of CREB and the coactivators CBP and CRTCs $(45,61)$. The cAMPmediated upregulation of PEPCK and NR4A2 gluconeogenic genes $(41,42,63)$ was almost erased upon SLU7 knockdown in cultured cells. Importantly, SLU7 was essential for the recruitment of CBP and RNA-Pol II to cAMP-responsive gene promoters, in which SLU7 was also detected, and was found to interact with P-CREB, CBP, and RNA-Pol II. cAMP signaling activates gene transcription by promoting $\mathrm{P}-\mathrm{CREB}$ and its association with coactivators like CBP, which in turn interact with components of the RNA-Pol II complex facilitating transcription (45). However, it has become apparent that the interaction of P-CREB with its coactivators would be too weak to drive gene activation per se, and that additional partners are required for stable and productive recruitment (41). In this context, we could be looking at a novel cellular function of SLU7 not related in principle to its role as splicing factor, but as a necessary component of the cAMP signaling pathway at the level of transcriptional complex assembly and target gene regulation. This functional diversity is not without precedent among splicing factors; for instance, hnRNPs have been identified as transcription regulators and coactivators for a number of genes (64), while NONO (also known as p54nrb) has also been reported to be a necessary component of cAMP-CREB-mediated gene transcription (42). SLU7 may thus be regarded as a new coactivator in the CAMP-CREB pathway regulating gluconeogenic gene expression. Importantly, the interaction between SLU7 and cAMP signaling might have implications exceeding the regulation of gluconeogenesis, as the cAMP pathway has also been involved in the maturation of the hepatic lineage and the expression of liverenriched genes (65). Our current observations of the strong effects of SLU7 on cAMP-CREB-regulated gene promoters, as well as its critical influence on $H N F 4 \alpha$ P1 and P2 promoter activity, suggest that transcriptional regulation would be a major biological role of this splicing factor. Future studies will need to delineate the mechanisms contributing to the recruitment of SLU7 to specific gene promoters and to identify the critical SLU7-interacting factors participating in the formation of transcriptional regulatory complexes. 
According to the significant roles played by SLU7 in the liver, regulation of its expression and availability must be tightly controlled processes. Previous studies demonstrated the nucleocytoplasmic shuttling of SLU7 protein in response to cellular stress $(1,52)$, and we also described how SLU7 transcription is repressed in hepatocytes by amphiregulin, a ligand of the epidermal growth factor receptor $(2,24)$. Here, we showed that hepatic SLU7 expression could be modulated by nutritional signals, being induced upon fasting and downregulated after feeding. Our in vitro findings demonstrated that cAMP stimulated expression of SLU7, whereas insulin exerted a negative effect on its transcription. These findings may suggest that, in vivo, the nutritional regulation of SLU7 expression could be mediated by glucagon and insulin signaling. The impaired postprandial downregulation of $\operatorname{Slu} 7$ transcription in mice fed a HFD would be consistent with the loss of insulin sensitivity that developed in this model. Nevertheless, further studies are warranted to fully understand the mechanisms underlying regulation of SLU7 gene expression in normal and diseased liver.

In summary, our current observations identified SLU7 as an essential mediator in the preservation of hepatocellular identity. Our finding that downregulation of SLU7 expression triggered a proliferative phenotype in healthy liver further emphasizes its pivotal role in the homeostasis of the organ. The loss of SLU7 expression occurring in the cirrhotic and preneoplastic liver (24) may be of high pathogenic relevance, contributing to the progressive deterioration of liver function up to HCC development. Further characterization of the mechanisms controlling SLU7 gene expression and its cellular targets may provide new avenues for therapeutic intervention in chronic liver disease.

\section{Methods}

Further information can be found in Supplemental Methods and Supplemental Tables 3 and 4.

RNAi. Human SLU7-specific siRNA (siSLU7) and the control siRNA (siGL) were from Sigma-Aldrich (24). Transfections were performed using Lipofectamine RNAiMAX reagent (Invitrogen) following the manufacturer's instructions. Silencing was confirmed by qPCR and Western blot.

9 siRNAs targeting mouse $\operatorname{Slu} 7$ were tested in vitro for their capacity to inhibit SLU7 expression. The 2 best performing sequences (siSLU7-767, TGGGCAGAATTTCGACTCTAA; siSLU7-723, GAGGATGAAGACGAAGACAAA) and a siRNA targeting Renilla (CAGGAATTATAATGCTTATCT), used as negative control, were cloned as shRNAs in the context of miR-30 (66). The 3 miR-shRNAs cassettes were subcloned in adenoassociated viral vectors (AAV8) flanked by AAV2 WT inverted terminal repeats, and under the regulation of a chimeric liver-specific promoter composed of the human $\alpha 1$-antitrypsin promoter (AAT) with regulatory sequences from the albumin enhancer (Eal) (67). The $2 \mathrm{AAV}$-shSLU7 vectors performed similarly in the in vivo experiments; the results presented correspond to siSLU7-767.

Production and purification of AAVs. To generate the AAV-SLU7 virus, mouse SLU7 cDNA was cloned in the AAV8 vector as described above. All rAAV8 viruses were produced by polyethylenimine-mediated (PEImediated) cotransfection of AAV8-miR-shRNAs or AAV8-SLU7 vectors with pDP8.ape plasmid (PlasmidFactory GmbH \& Co.) in HEK-293 cells (68). Cells were harvested 72 hours after transfection, and virus was released from the cells by 3 rounds of freeze-thawing. Crude lysate from all batches was then treated with RNAse and DNAse for 1 hour at $37^{\circ} \mathrm{C}$ and then kept at $-80^{\circ} \mathrm{C}$ until purification, which was performed by iodixanol gradients as described previously (69). The purified batches were concentrated and diafiltrated by cross-flow filtration with a molecular mass cutoff of $400 \mathrm{kDa}$. Batches were then concentrated further by passage through Centricon tubes (YM-100; Millipore) to a final concentration of $1 \times 10^{12} \mathrm{vg} / \mathrm{ml}$, as determined by qPCR. Finally, viral batches were filtered (pore size, $0.22 \mathrm{~mm}$ ) and stored at $-80^{\circ} \mathrm{C}$.

Animal experiments. C57BL/ 6 male mice (Harlan) were used. 16-week-old mice were injected i.v. with rAAVs $\left(1 \times 10^{11} \mathrm{pfu}\right)$. Experiments were performed 2-3 weeks after AAV injection with at least 5 animals per group and were repeated at least twice.

Mice were maintained under fed conditions with regular chow or fasted for 4 hours or overnight. In some experiments, after overnight fasting, mice were refed with regular chow diet for 4 hours. At sacrifice, blood was collected, and livers were weighed, snap-frozen in liquid nitrogen, and paraffin embedded. In some experiments, mice were fed a HFD (D12451 OpenSource Diets) for 5 months before fasting overnight and refeeding for 4 hours.

For the insulin tolerance test, after a 4-hour fast, mice received an i.p. injection of $1 \mathrm{U} / \mathrm{kg}$ insulin (Novo Nordisk Pharma) in saline. For the pyruvate tolerance test, after an overnight fast, animals received an i.p. injection of $2 \mathrm{~g} / \mathrm{kg}$ sodium pyruvate (Sigma-Aldrich) in saline. For glucagon challenge test, mice were fasted for 4 hours and i.p. injected with $100 \mu \mathrm{g} / \mathrm{kg}$ glucagon (Sigma-Aldrich). Blood glucose concentration was measured from the tail vein at different time points using an ACCUCHEK Aviva glucometer (Roche).

For hepatic lactate determination, frozen liver tissues were ground to powder in liquid nitrogen using mortar and pestle. Liver (approximately $30 \mathrm{mg})$ was homogenized $(1: 3 \mathrm{w} / \mathrm{v})$ with $1 \%$ perchloric acid on ice. The homogenate was centrifuged at $5,000 \mathrm{~g}$ for 10 minutes. The supernatant was collected, neutralized with $\mathrm{KOH}(1 \mathrm{~N})$, and centrifuged at $10,000 \mathrm{~g}$ for 10 minutes at $4^{\circ} \mathrm{C}$. Pellets were discarded, and supernatants were used for the determination of total lactate concentration on Roche/Hitachi Cobas system.

For hepatic glycogen measurement, about $100 \mathrm{mg}$ frozen liver tissue was ground to a powder, and $300 \mu \mathrm{l}$ of $30 \% \mathrm{KOH}$ was added. The homogenized tissue was heated to $100^{\circ} \mathrm{C}$ for 2 hours. To precipitate glycogen, 2 volumes of $95 \%$ ethanol were added, and samples were centrifuged at $1,500 \mathrm{~g}$ for 10 minutes. Pellets were resuspended in a minimal amount of distilled water and acidified to $\mathrm{pH} 3$ with $\mathrm{HCl}(5 \mathrm{~N})$. Glycogen was reprecipitated with ethanol, and pellets were dried and dissolved in hydrolysis buffer for analysis using Glycogen Assay Kit (BioVision).

${ }^{18}$ F-FDG PET. Liver glucose metabolism was assessed in vivo by PET with the glucose analog radiotracer 18 fluorodeoxyglucose $\left({ }^{18} \mathrm{~F}-\mathrm{FDG}\right)$. To improve liver uptake of ${ }^{18} \mathrm{~F}-\mathrm{FDG}$, mice were fasted overnight and warmed with a heating pad at $30^{\circ} \mathrm{C} 30$ minutes before the study and during the imaging period, and kept under continuous anesthesia with isoflurane ( $2 \%$ in $100 \% \mathrm{O}_{2}$ gas) (70). PET scans were obtained in a smallanimal dedicated imaging tomograph (Mosaic; Philips), with an axial and transaxial field of view of 11.9 and $12.8 \mathrm{~cm}$, respectively, and a full width at half-maximum resolution of $2.1 \mathrm{~mm}$. On the day of study, mice were placed prone on the PET scanner. Then, ${ }^{18} \mathrm{~F}-\mathrm{FDG}(7.5 \pm 0.5 \mathrm{MBq})$ was injected i.v. trough the tail vein simultaneously to the beginning of a list mode study of 30 minutes. 15 -frame dynamic sinograms were created $(2 \times 15 \mathrm{~s}, 7 \times 30 \mathrm{~s}, 1 \times 60 \mathrm{~s}, 1 \times 120 \mathrm{~s}, 1 \times 180 \mathrm{~s}, 2 \times 300 \mathrm{~s}$, and $1 \times 600 \mathrm{~s})$, and dynamic images were then reconstructed in a $128 \times 128$ matrix with a $1 \times 1 \times 1 \mathrm{~mm}^{3}$ voxel size using the 3D Ramla algorithm with 2 iterations, a relaxation parameter of 0.024 , and applying of dead time, decay, random, and scattering corrections. For assessment of liver ${ }^{18} \mathrm{~F}-\mathrm{FDG}$ uptake, all studies were exported and analyzed using PMOD software (PMOD Technologies Ltd.). Images were expressed in standardized uptake value (SUV) units, calculated as [tissue activity concentration $\left(\mathrm{Bq} / \mathrm{cm}^{3}\right) /$ injected dose $\left.(\mathrm{Bq})\right] \times$ body weight $(\mathrm{g})$. Because of the limited 
anatomical information of PET images, the volume of interest (VOI) of liver was drawn over an image of the first 2-4 minutes created from the dynamic study that reflects the vascular distribution of ${ }^{18} \mathrm{~F}-\mathrm{FDG}$. VOI was then transferred to an image of the last 10 minutes of the study (20-30 minutes after injection). From each VOI, mean SUV and maximum SUV were calculated.

$\mathrm{PH}$. Two-thirds $\mathrm{PH}$ and sham operations were performed as described previously (71) in control mice and mice injected 2 weeks prior with AAVshSLU7, AAV-SLU7, or control adenovirus (AAV-Ren). Animals were killed 3 or 34 hours after surgery, and liver samples were snap frozen.

Accession number. Microarray data were deposited in GEO (accession no. GSE54090).

Statistics. Statistical analysis was performed with Prism GraphPad software. Data are represented as mean \pm SEM. Normally distributed data were compared among groups using 2-tailed Student's $t$ test. Non-normally distributed data were analyzed using the Mann-Whitney test. Correlation was calculated with the Spearman test. A $P$ value less than 0.05 was considered significant.

Study approval. All animal studies were approved and performed in accordance with guidelines from the Ethics Committee for Animal Testing of the University of Navarra. This study was approved by the Human Research Review Committee of the University of Navarra, and written informed consent was received from participants prior to inclusion in the study.

\section{Acknowledgments}

We thank L. Guembe (CIMA, University of Navarra, Pamplona, Spain) for technical support with immunohistochemical analysis. This work was supported by the agreement between FIMA and the "UTE project CIMA"; RTICC-RD06 00200061 (to C. Berasain and M.A. Ávila); CIBEREhd (to I. Uriarte, J. Prieto, C. Berasain, and M.A. Ávila); and FIS PI10/02642, PI13/00359, PI10/00038, and PI13/00385 (to C. Berasain and M.A. Ávila) from Instituto de Salud Carlos III. M. Elizalde was supported by a fellowship from Gobierno de Navarra. R. Urtasun and M.U. Latasa were supported by a "Torres Quevedo" and a "Ramón y Cajal" contract from Ministerio de Educación, respectively. O. García-Irigoyen was supported by a FPU fellowship from Ministerio de Educación, Cultura y Deporte, Spain.

Received for publication November 20, 2013, and accepted in revised form March 28, 2014.

Address correspondence to: Matías A. Ávila and Carmen Berasain, Division of Hepatology and Gene Therapy, CIMA, Avda. Pio XII, n55. 31008 Pamplona, Spain. Phone: 34.948.194700; Fax: 34.948.194717; E-mail: maavila@unav.es (M.A. Ávila), cberasain@unav.es (C. Berasain).
1. Costa RH, Kalinichenko VV, Holterman A-XL, Wang X. Transcription factors in liver development, differentiation, and regeneration. Hepatology. 2003;38(6):1331-1347

2. Blachier M, Leleu H, Peck-Radosavljevic M, Valla D-C, Roudot-Thoraval F. The burden of liver disease in Europe: a review of available epidemiological data. J Hepatol. 2013;58(3):593-608.

3. Michalopoulos GK. Principles of liver regeneration and growth homeostasis. Compr Physiol. 2013;3(1):485-513.

4. Hernandez-Gea V, Friedman SL. Pathogenesis of liver fibrosis. Annu Rev Pathol. 2011;6:425-456.

5. Avila MA, et al. Reduced mRNA abundance of the main enzymes involved in methionine metabolism in human liver cirrhosis and hepatocellular carcinoma. J Hepatol. 2000;33(6):907-914.

6 . Okabe $\mathrm{H}$, et al. Genome-wide analysis of gene expression in human hepatocellular carcinomas using cDNA microarray: identification of genes involved in viral carcinogenesis and tumor progression. Cancer Res. 2001;61(5):2129-2137.

7. Berasain C, et al. Expression of Wilms' tumor suppressor in the liver with cirrhosis: relation to hepatocyte nuclear factor 4 and hepatocellular function. Hepatology. 2003;38(1):148-157.

8. Perugorría MJ, et al. Wilms' tumor 1 gene expression in hepatocellular carcinoma promotes cell dedifferentiation and resistance to chemotherapy. Cancer Res. 2009;69(4):1358-1367.

9 . Becker D, et al. Genetic signatures shared in embryonic liver development and liver cancer define prognostically relevant subgroups in HCC. Mol Cancer. 2012;11:55.

10. Behnke M, Reimers M, Fisher R. The expression of embryonic liver development genes in hepatitis $\mathrm{C}$ induced cirrhosis and hepatocellular carcinoma. Cancers (Basel). 2012;4(3):945-968.

11. Trevisani F, Cantarini MC, Wands JR, Bernardi $\mathrm{M}$. Recent advances in the natural history of hepatocellular carcinoma. Carcinogenesis. 2008; 29(7):1299-1305

12. Lerose R, Molinari R, Rocchi E, Manenti F, Villa E. Prognostic features and survival of hepatocellular carcinoma in Italy: impact of stage of disease. Eur J Cancer. 2001;37(2):239-245

13. Lee J-S, et al. A novel prognostic subtype of human hepatocellular carcinoma derived from hepatic progenitor cells. Nat Med. 2006;12(4):410-416.

14. Hoshida Y, et al. Gene expression in fixed tissues and outcome in hepatocellular carcinoma. $N$ Engl J Med. 2008;359(19):1995-2004.

15. Li J, Ning G, Duncan SA. Mammalian hepatocyte differentiation requires the transcription factor HNF-4a. Genes Dev. 2000;14(4):464-474.

16. Hayhurst GP, Lee YH, Lambert G, Ward JM, Gonzalez FJ. Hepatocyte nuclear factor $4 \alpha$ (nuclear receptor 2A1) is essential for maintenance of hepatic gene expression and lipid homeostasis. Mol Cell Biol. 2001;21(4):1393-1403.

17. Lazarevich NL, et al. Progression of HCC in mice is associated with a downregulation in the expression of hepatocyte nuclear factors. Hepatology. 2004; 39(4):1038-1047.

18. Sen S, Jumaa H, Webster NJG. Splicing factor SRSF3 is crucial for hepatocyte differentiation and metabolic function. Nat Commun. 2013;4:1336.

19. Yea $S$, et al. Ras promotes growth by alternative splicing-mediated inactivation of the KLF6 tumor suppressor in hepatocellular carcinoma. Gastroenterology. 2008;134(5):1521-1531.

20. Muñoz U, et al. Hepatocyte growth factor enhances alternative splicing of the Kruppel-like factor 6 (KLF6) tumor suppressor to promote growth through SRSF1. Mol Cancer Res. 2012; 10(9):1216-1227.

21. Chettouh $\mathrm{H}$, et al. Mitogenic insulin receptor-A is overexpressed in human hepatocellular carcinoma due to EGFR-mediated dysregulation of RNA splicing factors. Cancer Res. 2013;73(13):3974-3986.

22. Berasain C, et al. Impairment of pre-mRNA splicing in liver disease: mechanisms and consequences. World J Gastroenterol. 2010;16(25):3091-3102.

23. Reed R. Mechanisms of fidelity in pre-mRNA splicing. Curr Opin Cell Biol. 2000;12(3):340-345.

24. Castillo J, et al. Amphiregulin induces the alternative splicing of p73 into its oncogenic isoform DeltaEx2p73 in human hepatocellular tumors. Gastroenterology. 2009;137(5):1805-15.e1-4

25. Levine AJ, Puzio-Kuter AM. The control of the metabolic switch in cancers by oncogenes and tumor suppressor genes. Science. 2010;330(6009):1340-1344.

26. Ward PS, Thompson CB. Metabolic reprogramming: a cancer hallmark even warburg did not anticipate. Cancer Cell. 2012;21(3):297-308.

27. Hanahan D, Weinberg RA. Hallmarks of cancer: the next generation. Cell. 2011;144(5):646-674.

28. Lareau LF, Inada M, Green RE, Wengrod JC, Brenner SE. Unproductive splicing of SR genes associated with highly conserved and ultraconserved DNA elements. Nature. 2007;446(7138):926-929.

29. Samanez CH, et al. The human hepatocyte cell lines IHH and HepaRG: models to study glucose, lipid and lipoprotein metabolism. Arch Physiol Biochem. 2012;118(3):102-111.

30. Lu SC, Mato JM. S-adenosylmethionine in liver health, injury, and cancer. Physiol Rev. 2012; 92(4):1515-1542.

31. Ariel I, et al. The product of the imprinted $\mathrm{H} 19$ gene is an oncofetal RNA. Mol Pathol. 1997;50(1):34-44.

32. Madec S, et al. CYP4F3B expression is associated with differentiation of HepaRG human hepatocytes and unaffected by fatty acid overload. Drug Metab Dispos. 2011;39(10):1987-1996.

33. Santamaría M, et al. Nuclear $\alpha 1$-antichymotrypsin promotes chromatin condensation and inhibits proliferation of human hepatocellular carcinoma cells. Gastroenterology. 2013;144(4):818-828.e4.

34. Hai T, Wolfgang CD, Marsee DK, Allen AE, Sivaprasad U. ATF3 and stress responses. Gene Expr. 1999;7(4-6):321-335.

35. Derdak Z, et al. Inhibition of p53 attenuates steatosis and liver injury in a mouse model of non-alcoholic fatty liver disease. J Hepatol. 2013;58(4):785-791.

36. Nordlie RC, Foster JD, Lange AJ. Regulation of glucose production by the liver. Annu Rev Nutr. 1999; 19:379-406.

37. Frasca F, et al. Insulin receptor isoform A, a newly recognized, high-affinity insulin-like growth factor II receptor in fetal and cancer cells. Mol Cell Biol. 1999;19(5):3278-3288.

38. Yuneva MO, et al. The metabolic profile of tumors depends on both the responsible genetic lesion and tissue type. Cell Metab. 2012;15(2):157-170.

39. Panasyuk G, et al. PPAR $\gamma$ contributes to PKM 2 and HK2 expression in fatty liver. Nat Commun. 2012; 3:672-679.

40. Torres-Padilla ME, Fougère-Deschatrette C, Weiss $\mathrm{MC}$. Expression of $\mathrm{HNF} 4 \alpha$ isoforms in mouse liver development is regulated by sequential promoter usage and constitutive $3^{\prime}$ end splicing. Mech Dev. 
2001;109(2):183-193

41. Zhang X, et al. Genome-wide analysis of cAMPresponse element binding protein occupancy, phosphorylation, and target gene activation in human tissues. Proc Natl Acad Sci U S A. 2005; 102(12):4459-4464.

42. Amelio AL, et al. A coactivator trap identifies NONO (p54nrb) as a component of the cAMPsignaling pathway. Proc Natl Acad Sci U S A. 2007; 104(51):20314-20319.

43. Leahy P. CREB binding protein coordinates the function of multiple transcription factors including nuclear factor I to regulate phosphoenolpyruvate carboxykinase (GTP) gene transcription. J Biol Chem. 1999;274(13):8813-8822.

44. Duong DT, Waltner-Law ME, Sears R, Sealy L, Granner DK. Insulin inhibits hepatocellular glucose production by utilizing liver-enriched transcriptional inhibitory protein to disrupt the association of CREB-binding protein and RNA polymerase II with the phosphoenolpyruvate carboxykinase gene promoter. J Biol Chem. 2002;277(35):32234-32242.

45. Altarejos JY, Montminy M. CREB and the CRTC co-activators: sensors for hormonal and metabolic signals. Nat Rev Mol Cell Biol. 2011;12(3):141-151.

46. Raghow R, Yellaturu C, Deng X, Park EA, Elam MB. SREBPs: the crossroads of physiological and pathological lipid homeostasis. Trends Endocrinol Metabol. 2008;19(2):65-73.

47. Haas JT, et al. Hepatic insulin signaling is required for obesity-dependent expression of SREBP-1C mRNA but not for feeding-dependent expression. Cell Metab. 2012;15(6):873-884.

48. Wan M, et al. A noncanonical, GSK3-independent pathway controls postprandial hepatic glycogen deposition. Cell Metab. 2013;18(1):99-105.

49. Khamzina L, Gruppuso PA, Wands JR. Insulin signaling through insulin receptor substrate 1 and 2 in normal liver development. Gastroenterology. 2003;125(2):572-585.

50. Briançon N, Weiss MC. In vivo role of the HNF4 $\alpha$
AF-1 activation domain revealed by exon swapping. EMBO J. 2006;25(6):1253-1262.

51. Chua K, Reed R. The RNA splicing factor hSlu7 is required for correct $3^{\prime}$ splice-site choice. Nature. 1999;402(6758):207-210.

52. Shomron N, Alberstein M, Reznik M, Ast G. Stress alters the subcellular distribution of hSlu7 and thus modulates alternative splicing. J Cell Sci. 2005;118(pt 6):1151-1159.

53. Nakhei H, Lingott A, Lemm I, Ryffel GU. An alternative splice variant of the tissue specific transcription factor HNF $4 \alpha$ predominates in undifferentiated murine cell types. Nucleic Acids Res. 1998; 26(2):497-504.

54. Tanaka T, et al. Dysregulated expression of P1 and P2 promoter-driven hepatocyte nuclear factor$4 \alpha$ in the pathogenesis of human cancer. J Pathol. 2006;208(5):662-672.

55. Rhee J, et al. Regulation of hepatic fasting response by PPAR $\gamma$ coactivator- $1 \alpha$ (PGC-1): requirement for hepatocyte nuclear factor $4 \alpha$ in gluconeogenesis. Proc Natl Acad Sci U S A. 2003;100(7):4012-4017.

56. Bonzo JA, Ferry CH, Matsubara T, Kim J-H, Gonzalez FJ. Suppression of hepatocyte proliferation by hepatocyte nuclear factor $4 \alpha$ in adult mice. J Biol Chem. 2012;287(10):7345-7356.

57. Hanse EA, et al. Cyclin D1 inhibits hepatic lipogenesis via repression of carbohydrate response element binding protein and hepatocyte nuclear factor 4 $\alpha$. Cell Cycle. 2012;11(14):2681-2690.

58. Walesky C, et al. Hepatocyte nuclear factor $4 \alpha$ deletion promotes diethylnitrosamine-induced hepatocellular carcinoma in rodents. Hepatology. 2013; 57(6):2480-2490

59. David CJ, Chen M, Assanah M, Canoll P, Manley JL. HnRNP proteins controlled by c-Myc deregulate pyruvate kinase mRNA splicing in cancer. Nature. 2010;463(7279):364-368.

60. Webster NJ, et al. Differential effects of Wilms tumor WT1 splice variants on the insulin receptor promoter. Biochem Mol Med. 1997;62(2):139-150.
61. Jitrapakdee S. Transcription factors and coactivators controlling nutrient and hormonal regulation of hepatic gluconeogenesis. Int J Biochem Cell Biol. 2012;44(1):33-45.

62. Allen-Jennings AE, Hartman MG, Kociba GJ, Hai T. The roles of ATF3 in glucose homeostasis. A transgenic mouse model with liver dysfunction and defects in endocrine pancreas. J Biol Chem. 2001; 276(31):29507-29514.

63. Pei L, et al. NR4A orphan nuclear receptors are transcriptional regulators of hepatic glucose metabolism. Nat Med. 2006;12(9):1048-1055.

64. Lau JS, et al. Heterogeneous nuclear ribonucleoproteins as regulators of gene expression through interactions with the human thymidine kinase promoter. J Cell Biochem. 2000;79(3):395-406.

65. Ogawa S, et al. Three-dimensional culture and cAMP signaling promote the maturation of human pluripotent stem cell-derived hepatocytes. Development. 2013;140(15):3285-3296.

66. Zeng Y, Cai X, Cullen BR. Use of RNA polymerase II to transcribe artificial microRNAs. Meth Enzymol. 2005;392:371-380.

67. Kramer M. In vitro and in vivo comparative study of chimeric liver-specific promoters. Mol Ther. 2003;7(3):375-385.

68. Gil-Fariña I, et al. IL12-mediated liver inflammation reduces the formation of AAV transcriptionally active forms but has no effect over preexisting AAV transgene expression. PLoS One. 2013; 8(7):e67748.

69. Zolotukhin S, et al. Recombinant adeno-associated virus purification using novel methods improves infectious titer and yield. Gene Ther. 1999; 6(6):973-985

70. Fueger BJ, et al. Impact of animal handling on the results of 18 F-FDG PET studies in mice. J Nucl Med. 2006;47(6):999-1006.

71. Berasain C, et al. Amphiregulin: an early trigger of liver regeneration in mice. Gastroenterology. 2005;128(2):424-432. 